\title{
Revisión sistemática sobre la salud mental en el contexto COVID-19
}

\author{
Systematic review on mental health in the context of \\ COVID-19
}

Carmen N. León ${ }^{1} \quad$ (iD Edith S. Olivera ${ }^{2}$

1 Universidad César Vallejo. Lima, Perú

${ }^{2}$ Universidad Le Cordon Bleu. Lima, Perú

Recibido: 06/12/2021 Revisado: 13/12/2021 Aceptado: 02/01/2022 Publicado: 15/01/2022

\section{RESUMEN:}

La coyuntura actual permitió visualizar el impacto contraproducente para atender la salud mental de las personas. Por un lado, trabajadores, estudiantes y personal sanitario requirió de atención para el tratamiento de ciertos malestares psicológicos. Inclusive los nosocomios se vieron afectados en sus programas de prevención y promoción de la salud mental. Por ello, esta investigación pretender analizar los aportes sobre la salud mental durante la pandemia. La metodología que se utilizó fue la revisión sistemática y la aplicación del diagrama de flujo PRISMA para la selección de artículos indizados. Los principales aportes han mostrado que la salud mental sufrió cambios en el tratamiento y seguimiento de pacientes. Además, los grupos afectados han sido los trabajadores, estudiantes de educación regular y superior, y el personal sanitario. Dichos grupos se vieron afectados directamente por el aislamiento, lo cual produjo un desequilibrio de su salud mental. Entre las primordiales consecuencias se vio un incremento de la ansiedad, depresión y estrés. Por último, las estrategias empleadas sugieren el empleo de actividades físicas, cambio de hábitos y establecer cronogramas para el control de actividades. Palabras clave: Salud mental, bienestar mental, ansiedad, depresión, pandemia, covid-19.

\section{ABSTRACT:}

The current situation made it possible to visualize the counterproductive impact on attending to people's mental health. On the one hand, workers, students, and health personnel required attention for the treatment of certain psychological discomforts. Even nosocomios were affected in their mental health prevention and promotion programs. Therefore, this research aims to analyze the contributions on mental health during the pandemic. The methodology used was the systematic review, in addition to the PRISMA flowchart was applied for the selection of indexed articles. The main contributions have shown that mental health underwent changes in the treatment and follow-up of patients. In addition, the groups affected have been workers, students in regular and higher education, and health workers. These groups were directly affected 
by isolation, resulting in an imbalance in their mental health. Among the main consequences was an increase in anxiety, depression and stress. Finally, the strategies used suggest the use of physical activities, changing habits and establishing schedules for the control of activities. Keywords: Mental health, mental well-being; anxiety, depression, pandemic, covid-19.

\section{INTRODUCCIÓN}

Debido a la incontrolable propagación del Covid-19 y sus efectos en la salud pública, el 11 de marzo del 2020 la Organización Mundial de la Salud (OMS, 2020) la declaró como pandemia. Ante esto, diferentes países decidieron aplicar políticas para evitar el contagio, siendo una de ellas el aislamiento social obligatorio o cuarentena. Esta medida fue tomada con el fin de evitar el colapso de centros de salud y atenuar el número de fallecimientos. A inicios de la pandemia, se priorizaron los estudios sobre salud física, dejando de lado los trabajos en el campo de la salud mental. Luego se comenzó a identificar problemas referidos a la ansiedad, estrés, violencia y depresión, los cuales fueron las manifestaciones de la restricción social, que prohibió las reuniones sociales y los eventos recreativos. Inclusive, a este problema se sumó la falta de conocimientos e investigaciones sobre cómo abordar el aislamiento social y sus efectos en la estabilidad mental de la gente.

Las personas se vieron afectadas por un repentino aislamiento, y entre todos los pacientes con trastornos del ánimo, como la ansiedad, mostraron ser un grupo con mayor vulnerabilidad debido a su tendencia a la inestabilidad cognitiva propio del padecimiento de dicha patología. De allí la necesidad de disponer de estudios (que incrementen el acervo bibliográfico) de las consecuencias resultantes por el aislamiento. En otras palabras, la finalidad es producir conocimiento sobre las consecuencias que producirían situaciones similares en el futuro.
Han sido muchos las personas que han llevado terapia psicológica y psiquiátrica durante la pandemia. Se incrementaron con intensidad los cuadros de ansiedad y depresión en los diferentes grupos de edades. Entre ellos se vieron más afectados las personas que iniciaron sus trabajos de forma remota, estudiantes del nivel básico y superior, adultos mayores con enfermedades médicas (Barrera et al., 2019). En la mayoría de los casos se recomendó la atención social y cercana. Por un lado, los adolescentes fueron el grupo de mayor contacto con lo digital, lo cual pudo beneficiar su proceso de enseñanza virtual, sin embargo, estuvieron afectados por la ansiedad debido a que el uso temprano de redes sociales (Facebook, Whatsapp, entre otros) genera ansiedad generalizada. (Tresáncoras et al., 2017). Los adultos que laboraban presencialmente también tuvieron dificultades dado que el aislamiento produjo que toda actividad se realice en casa; y en otros casos, incrementó el desempleo. Los adultos mayores con enfermedades médicas también tuvieron dificultades; por ejemplo, aquellos pacientes con patologías cardiacas que se encuentran vulnerables a sufrir episodios depresivos (Medina et al., 2021). Lacruz-Gascón et al. (2019) también sustenta que el estado anímico tiene el poder suficiente para deteriorar la salud mental y física; es decir, emociones negativas del paciente pueden producir que la enfermedad empeore o que surja una comorbilidad somática o psiquiátrica (Herrera et al., 2021). Y a la vez debe de tomarse en cuenta que al igual que los adultos mayores, adultos jóvenes y adolescentes, los niños también pueden 
verse afectados por la ansiedad y depresión.

Sin embargo, la bibliografía sobre la salud mental se viene incrementando sin que exista un estudio que recopile estos aportes y pueda resumir la información en un solo documento. La necesidad de conocer la situación de la pandemia en la salud mental se ha vuelto una prioridad. Por ello, el principal objetivo de este estudio es analizar los aportes sobre la salud mental durante el contexto de pandemia a raíz del covid-19. De esta forma, se agrupó la información para facilitar la lectura sobre el contenido del tema; de igual forma, se profundizó sobre los factores psicológicos de mayor atención y riesgo.

\section{MATERIALES Y MÉTODOS}

Se efectuó la revisión sistemática como método de investigación y se aplicó la declaración PRISMA (Urrutía y Bonfill, 2011) para la recopilación y filtraje de la información. Para cumplir los estándares de calidad se consideró el uso plenamente de artículos científicos que hayan sido publicados en revistas indexadas de revisión por pares. La búsqueda de información se estableció dentro de bases de datos de impacto como: Scielo, Dialnet, Web of Science y SCOPUS

Por otro lado, para la búsqueda de información se utilizaron las siguientes palabras claves: "salud mental", "covid-19", "ansiedad", “depresión”, "estrés”, "bienestar mental”. Además, se consideró que todo artículo de investigación esté en español. De esa forma se delimitó la información en las respectivas bases de datos. Por consiguiente, la información obtenida se almacenó en un Excel con todos los datos necesarios. No obstante, para poseer un sistema de flujo adecuado se utilizó el proceso de PRISMA como se observa en la figura 1.

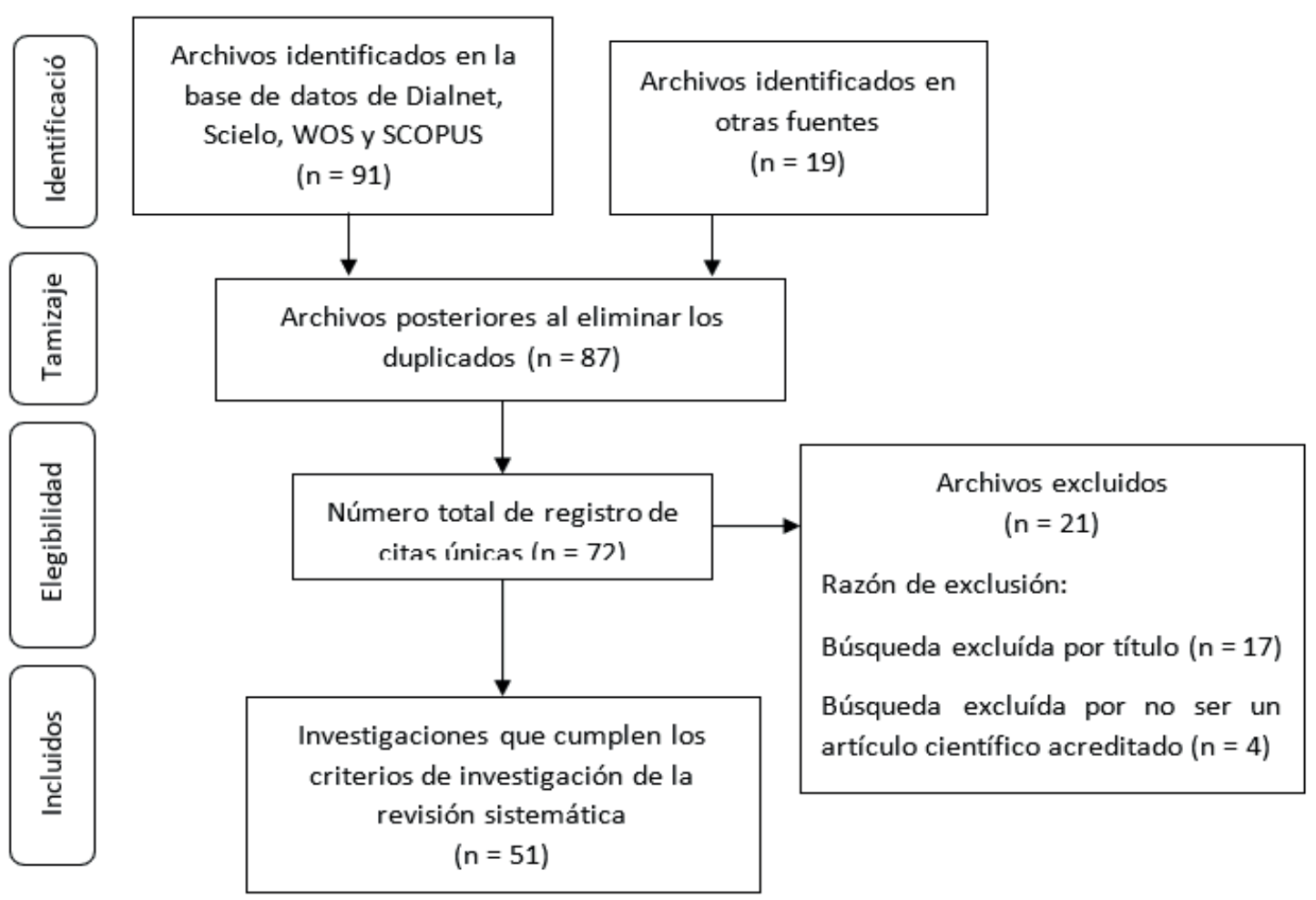

Figura 1. Diagrama de flujo (PRISMA) 
Inicialmente, se obtuvieron un total de 91 artículos de investigación sobre el tema encontrados en las bases de datos de búsqueda, por otro lado, también se encontraron artículos mediante otro tipo de búsqueda (indirecta) siendo 19 investigaciones. En total se tuvieron 72 artículos totales de los cuales se excluyeron después de eliminar los duplicados. Posterior- mente se analizó su elegibilidad y se excluyeron 17 por el título irrelevante al tema, y 4 por no mostrar seriedad de la revista. Finalmente se obtuvieron 51 artículos de investigación con el contenido temático y seriedad que se requirieron. Los artículos incluidos dentro del estudio se pueden apreciar en la tabla 1.

Tabla 1. Artículos consultados sobre la salud mental en el contexto Covid-19

\begin{tabular}{|c|c|c|c|}
\hline $\mathbf{N}$ & Autores & Tipo de estudio & Base de datos \\
\hline 1 & Acosta-Quiroz y Iglesias-Osores (2020) & Carta al editor & Web of Science \\
\hline 2 & Alarcón-Guzmán (2020) & Artículo de opinión & Web of Science \\
\hline 3 & Alomo et al. (2020) & Epidemiológico & Dialnet \\
\hline 4 & Álvarez-Villaseñor et al. (2020) & Asociativo & Scielo \\
\hline 5 & Bakr et al. (2020) & Epidemiológico & Scopus \\
\hline 6 & Becerra y Ybaseta (2020) & Asociativo & Scielo \\
\hline 7 & Bueno-Notivol et al. (2021) & Revisión sistemática & Scopus \\
\hline 8 & Caetano et al. (2020) & Revisión sistemática & Scielo \\
\hline 9 & Correa et al. (2020) & Carta al editor & Scopus \\
\hline 10 & De la Rosa et al. (2020) & Revisión sistemática & Dialnet \\
\hline 11 & Dos Santos et al. (2021) & Artículo de opinión & Scopus \\
\hline 12 & Dosil et al. (2020) & Asociativo & Scopus \\
\hline 13 & Eidman et al. (2020) & Asociativo & Dialnet \\
\hline 14 & Faro et al. (2020) & Artículo de revisión & Scielo \\
\hline 15 & Galindo-Vazquez et al. (2020) & Asociativo & Dialnet \\
\hline 16 & García-Iglesias et al. (2020) & Revisión sistemática & Scopus \\
\hline 17 & Gómez (2020) & Descriptivo & Scopus \\
\hline 18 & González-Jaimes et al. (2020) & Observacional & Scielo \\
\hline 19 & González-Rodríguez y Labad (2020) & Artículo de reflexión & Scopus \\
\hline 20 & Hernández-Rodríguez (2020) & Transversal & Scielo \\
\hline 21 & Herrera et al. (2021) & Descriptivo & Scopus \\
\hline 22 & Huarcaya-Victoria (2020) & Descriptivo & Scopus \\
\hline 23 & Inchausti et al. (2020) & Artículo de opinión & Scopus \\
\hline 24 & Juaréz-García (2020) & Artículo de revisión & Scielo \\
\hline 25 & Leiva et al. (2020) & Fenomenológico & Scielo \\
\hline 26 & López y López (2021) & Artículo de opinión & Dialnet \\
\hline 27 & Lorenzo et al. (2021) & Observacional & Scielo \\
\hline 28 & Lozano-Vargas (2020) & Descriptivo & Web of Science \\
\hline 29 & Mazza et al. (2020) & Epidemiológico & Scopus \\
\hline 30 & Medina et al. (2021) & Asociativo & Scopus \\
\hline 31 & Merino-Navarro y Díaz-Periánez (2021) & Descriptivo & Scopus \\
\hline 32 & Motrico et al. (2020) & Artículo de opinión & Scopus \\
\hline 33 & Muñoz et al. (2020) & Fenomenológico & Scopus \\
\hline 34 & Ochoa et al. (2020) & Descriptivo & Dialnet \\
\hline 35 & Ornell et al. (2020) & Descriptivo & Scopus \\
\hline 36 & Ozamiz-Etxebarria et al. (2020) & Descriptivo & Scielo \\
\hline 37 & Pech-Puebla et al. (2021) & Asociativo & Scopus \\
\hline 38 & Pérez et al. (2021) & Asociativo & Dialnet \\
\hline 39 & Presa-García et al. (2020) & Fenomenológico & Scielo \\
\hline 40 & Ramírez-Ortiz et al. (2020) & Artículo de reflexión & Web of Science \\
\hline
\end{tabular}




\begin{tabular}{llll}
41 & Reis et al. (2020) & Carta al editor & Scopus \\
42 & Ribot et al. (2020) & Revisión sistemática & Scopus \\
43 & Robles-Sánchez (2020) & Artículo de opinión & Scopus \\
44 & Rodríguez et al. (2020) & Revisión sistemática & Scopus \\
45 & Sánchez (2021) & Artículo de revisión & Scopus \\
46 & Sandín et al. (2020) & Descriptivo & Web of Science \\
47 & Saravia-Bartra et al. (2020) & Asociativo & Scielo \\
48 & Torres-Muñoz et al. (2020) & Revisión sistemática & Scopus \\
49 & Urzúa et al. (2020) & Revisión sistemática & Scielo \\
50 & Valero et al. (2020) & Artículo de revisión & Dialnet \\
51 & Ybaseta-Medina y Becerra-Canales (2020) & Artículo de opinión & Scielo \\
\hline
\end{tabular}

\section{RESULTADOS Y DISCUSIÓN}

\section{Salud mental en la población general}

Las medidas de restricción social que se impusieron afectaron el aspecto moral y emocional, lo cual tuvo implicancias en el aspecto laboral, la eficacia, competencia y lo intelectual, lo que repercutió en la disminución de la productividad en el trabajo o en la educación (Ribot et al., 2020). Además, Ribot et al. (2020) estudió los factores que podrían causar daños psicológicos en las personas durante la pandemia; de esa forma las clínicas psicológicas -con la ayuda del personal de salud- tuvieron que realizar un trabajo intenso para ayudar a las personas que padecen de enfermedades psiquiátricas o que están propensas a adquirirlas. En España las investigaciones han demostrado que es la prevención de patologías anímicas, lo que reduce la probabilidad de contraer problemas emocionales; sin embargo, si la persona padece de estos problemas mentales se incrementaría la dificultad de solucionarlos (Roble-Sánchez, 2020; Sandín et al., 2020).

Por otro lado, luego de varios meses de la propagación del Covid-19 se encontró un aumento de patologías negativas como depresión, ansiedad, estrés, frustración, etc. Se observó mayor posibilidad de adquirir emociones negativas que positivas con el transcurso de los días en el periodo de aislamiento. Por la falta de la interacción social se incrementó el uso de redes sociales donde se difundía contenido que en gran parte carecía de sustento científico lo cual promovía un estado de alarma personal (estrés) y temor a lo que podría pasar (ansiedad) (Huarcaya-Victoria, 2020; Saravia-Bartra et al., 2020). Las personas empezaron a desarrollar distintos síntomas de ansiedad y problemas de convivencia por no tener una buena salud mental ni inteligencia emocional.

Asimismo, se reconoce que los diferentes malestares emocionales y la situación de aislamiento aumentaron la posibilidad de traumas y problemas psicológicos; motivo por el cual, los especialistas tuvieron que adaptarse a las circunstancias para tratar a pacientes con ansiedad y, de esa forma, prevenir o solucionar problemas causados por la pandemia (Leiva et al., 2020; Medina et al., 2021). También en el aspecto profesional de salud diversos médicos, enfermeros(as) y colaboradores cuya función está relacionada con la emergencia sanitaria actual, se vieron perjudicados emocional y psicológicamente ya que ellos están en la primera línea de trabajo y reciben diversos pacientes con complicaciones por este virus (Herrera et al., 2021; Merino-Navarro y Díaz-Periánez, 2020). Son quienes precisamente se encuentran propensos a contagiarse; por ese 
motivo se incrementaron los casos de ansiedad y síntomas falsos referidos al covid-19. Además, muestran preocupación de portar el virus y contagiar a sus familiares. Fueron muchos los profesionales que tuvieron que tomar terapia psicológica (Lozano-Vargas, 2020).

Entre los universitarios se observaron una gran cantidad de problemas mentales y emocionales a causa de esta pandemia. Debido a las noticias e informaciones falsas con la reciente propagación del virus, el pueblo estudiantil se alarmó y se incrementó la presencia de ansiedad, estrés, frustración, etc. En la educación básica, se vio una clara disminución del rendimiento académico debido a los problemas familiares, malhumor, ansiedad, entre otros. Los estudios registraron que los estudiantes poseían ansiedad elevada lo cual afectó al comportamiento académico y en el cuidado personal. Sin embargo, hubo un grupo que presentó ansiedad baja debido a que no mostraban interés en las medidas de precaución (Ornell et al., 2020). Por consiguiente, los estudiantes y profesores tuvieron que adaptarse a la educación virtual. Los docentes que no se encontraban familiarizados con la virtualidad pasaron episodios de tensión y ansiedad (Dos Santos et al., 2021).

En relación con las consecuencias por el efecto de las medidas tomadas frente al coronavirus en el aspecto psicológico de niños $\mathrm{y}$ adolescentes, se estima que quienes sufrían de afecciones previas, incluyendo psicopatologías, son los más vulnerables. Se registró una alta cifra de atenciones y hospitalizaciones médicas, debido que muchos adolescentes vieron afectada su salud mental durante el confinamiento social (González-Rodríguez y Labad, 2020; Sánchez, 2021). Aunque también, se ha podido observar una drástica baja en la ansie- dad y depresión presente en los adolescentes que sufrían acoso escolar (bullying o ciberbullying) al encontrarse lejos de sus agresores; lo cual puede demostrar en el ámbito académico, donde se han registrado alzas en sus notas, debido a que han tenido mayor concentración y apoyo dentro de sus hogares (Gómez, 2020).

Por otra parte, el temor a contraer la temible enfermedad ha inducido a muchas personas a tomar medidas de extremo autocuidado con el fin de evitar contagios. Como consecuencia, se ha comprobado el aumento de cuadros de ansiedad y depresión en las personas que llegaron a ser contagiadas, así como de problemas de estrés al verse discriminadas por estar contagiados (Galindo-Vázquez et al., 2020). Además, los suicidios y autolesiones se incrementaron en personas que se enfermaban de covid-19; al mismo tiempo, que los índices de depresión y estrés postraumático aumentaron en el confinamiento. Los rasgos marcados que evidenciaron predominancia de estados emocionales negativos fueron la tristeza, desolación, preocupación, entre otros (Lorenzo et al., 2021).

\section{Depresión}

Durante el tiempo transcurrido por el aislamiento social, se llegaron a reportar casos de depresión, por efecto de la soledad, frustración, desesperación e inutilidad que provocaron la falta de acercamiento a sus familiares que habitan en viviendas diferentes; así como la alteración del ciclo del sueño, al mantenerse mayor tiempo despierto durante la noche. Se ha determinado que la desregularización del sueño produce que las personas tengan pensamientos negativos intensificando las patologías anímicas como la depresión; inclusive, se muestran pensamientos recurrentes de suicidio (ideación suicida) (Sandín et al., 2020). 
Un estudio metanalítico demostró que la depresión se presentó en el 7,45 \% y el 48,30 $\%$ de personas evaluadas y la prevalencia colectiva frente fue del $25 \%$. La investigación muestra la comparación con una prevalencia global aproximada de depresión del 3,44\% en el año 2017, versus la prevalencia obtenida del $25 \%$, lo que manifiesta un impacto siete veces mayor, lo que sugiere que el COVID-19 fomentó una preocupación mayor en la salud mental de la población (Bueno-Notivol et al., 2020). Además, en los pacientes que se contagian con covid-19 aumenta la probabilidad de depresión, al mismo tiempo incrementa gravemente las emociones negativas al punto de producir distorsiones cognitivas (Hernández-Rodríguez, 2020). Por lo tanto, se requiere de diferentes especialistas psicológicos que apoyen en la prevención o el tratamiento de la depresión. Las personas que están al cuidado de un paciente con Covid-19 y desconocen cómo afrontar la enfermedad, terminan con signos de tristeza, estrés, impotencia, etc.

Para los pacientes depresivos, que necesitan de contactos sociales y una red de soporte, el cambio de la rutina social por el aislamiento puso en riesgo su salud mental. Se evidenció un incremento del cuadro de ansiedad y estrés, lo que impulsó la recurrencia de pensamientos negativos que pueden conllevar a la ideación suicida e inclusive al acto mismo. Por ello los aportes sobre la intervención del profesional en psicología debe ser constante y con nuevas estrategias para afrontar estos problemas del paciente depresivo en el aislamiento (Ozamiz-Etxebarria et al., 2020).

\section{Reacciones al estrés}

En el periodo de brote de fase inicial provocado por la pandemia, algunos estudios reportaron el incremento del estrés, es- pecialmente en los adultos mayores, quienes fueron los principales afectados dado que formaban parte del grupo con mayor tasa de mortalidad causado por el Covid-19. En la población joven y adultos, la sobrecarga de información a través de redes sociales era el principal factor del estrés, de igual modo, el alejamiento del contacto social contribuyó al incremento exponencial de problemas psicológicos como la ansiedad y estrés; lo que fue corroborado por los profesionales en la salud mental, quienes registraron un aumento en los casos de estrés, con mayor frecuencia los casos agudos, y ansiedad durante la pandemia (González-Jaimes et al., 2020).

Por otro lado, se encontró que otra de las causas del estrés ha sido la imposibilidad de realizar la rutina diaria durante la pandemia, lo que causó frustración y ansiedad. Además, la falta de contacto con sus familiares por el temor a contagiarlos fue un problema grave debido a que se restringió el diálogo cercano y los momentos recreativos (Ochoa et al., 2020). Un estudio en China, indicó que el $53 \%$ de la población tuvo un impacto psicológico a raíz de los contagios masivos; de los cuales, el $8 \%$ presentó niveles moderados y severos de estrés, lo que perjudicó al desarrollo de trastornos por comorbilidad (p.e. ansiedad, trastornos del sueño, pensamientos irracionales, depresión, ideación suicida, entre otros.) (Pech-Puebla et al., 2021; Ramírez-Ortiz et al., 2020).

En síntesis, la mayoría de los estudios sobre el estrés en el contexto de pandemia, señalan que se presenta en personas que frecuentemente se preocupan de la posibilidad de contagiarse. El segmento de la población con mayor riesgo de obtener estrés son los adultos mayores, pacientes psiquiátricos (ansiedad patológica, depresión) y personas con 
hábitos de socialización. Además, la presencia de estrés puede ser un signo de que otras enfermedades mentales están emergiendo.

\section{Ansiedad por la salud}

La ansiedad se manifiesta mediante expresiones cognitivas, físicas y conductuales, pero también ciertas personas suelen reflejar síntomas de ansiedad a través de las interpretaciones sobre una situación problemática. En el contexto de pandemia, pensar o imaginarse ser contagiado ha producido un incremento de rasgos de ansiedad, inclusive se manifiesta a un grado de somatizar ciertos pensamientos basado en los síntomas del virus (Bark et al., 2020). Todo este proceso de ansiedad ha traído como consecuencia determinados comportamientos compulsivos (exceso de limpieza, compra descontrolada de productos alimenticios o de limpieza, etc.). Por otro lado, a diferencia de aquellas personas que presentan síntomas de ansiedad, se encuentran quienes no perciben el contagio o transmisión del virus como un peligro (Mazza et al., 2020). Lo cual produce pensamientos acerca de una realidad inmune que reafirma que no se contagiarán porque la pandemia es una mentira (Motrico et al., 2020).

Entre las causas por las cuales se registraron aumentos en los casos de ansiedad durante el aislamiento social (Álvarez-Villaseñor et al., 2020), se ha identificado en primer lugar la necesidad de recaudar la mayor cantidad de alimentos y medicinas, así como el incremento de la incertidumbre sobre el presente y futuro de la vida laboral, social y familiar provocado por el largo periodo de aislamiento; y la preocupación de cuándo terminará la pandemia (De la Rosa et al., 2020).

Por lo tanto, es mediante el pensamiento de un futuro catastrófico o la incertidumbre por la cual se produce la ansiedad. Cuando una persona se imagina ser contagiado y perder la vida, es razonable su estado de alarma y de preocupación; sin embargo, cuando estos pensamientos sobrepasan lo regulado se necesitaría un tratamiento para disminuir el estado ansioso. En definitiva, la ansiedad ha sido un factor de impacto en la población mundial a raíz de la pandemia, y el incremento de casos se encuentra justificado por un pensamiento racional (preocupación) que se convierte en irracional (preocupación sin que haya pasado un hecho).

\section{Salud mental en los pacientes con enferme- dades mentales previas}

Durante el año 2020, la atención prestada a personas con enfermedades mentales se ha encontrado en segundo plano. La llegada de la pandemia los ha desplazado, más de lo que ya se encontraban dentro del sistema de salud. Los problemas mentales, en un escenario de pandemia, acarrea graves consecuencias, ya sean físicas (deterioro cognitivo) o psicológicas (depresión, ansiedad, entre otros) para los pacientes. Adicionalmente la problemática dentro de los centros hospitalarios psiquiátricos se ha incrementado en términos logísticos y de personal especializado, así como el establecimiento de límites para la implementación de un protocolo para evitar la propagación del virus (Huarcaya-Victoria, 2020).

El impacto de las medidas tomadas en el aspecto social y emocional ha tenido secuela, particularmente, en los adultos mayores que padecen algún trastorno o deterioro cognitivo, de igual modo hacia la población con enfermedades mentales. Esto es debido a que el aislamiento ha aumentado los niveles de ansiedad, depresión, estrés, entre otros; por ello es importante tener un control del impacto psicológico dentro de las poblaciones 
vulnerables causadas por el coronavirus, así como el deber de brindar información certera (Hernández-Rodríguez, 2020). El efecto dentro de esta población se refleja en diversos aspectos (emocional, social, etc.), por lo que resulta imprescindible que se tomen medidas para disminuir el impacto, velando así por la integridad mental de las personas (Ribot et al., 2020). Estos autores dan a comprender lo perjudicial que ha sido dejar de lado la atención de la salud mental. Muchos pacientes que padecen enfermedades mentales han sido puestas en segundo plano, debido a que no pueden tener un adecuado tratamiento.

Se ha demostrado que los pacientes con enfermedades mentales son más vulnerables a incrementar su patología a raíz de la pandemia. Para ello, se propuso intervenciones para reducir contagios y que el efecto a la salud mental sea menor, lo que incluyó campañas de soporte de salud mental y de información sobre el contagio del virus (Urzúa et al., 2020). Cabe recordar que las personas con condiciones psiquiátricas diagnosticadas ya se consideraban como una población en riesgo por la falta de políticas públicas que ayuden a mejorar su calidad de vida, bienestar psicológico y reducción de síntomas patológicos. Por eso mismo, la pandemia incrementó la necesidad de implementar nueva legislación para brindarles calidad de servicio a esta población (Ornell et al., 2020).

En España, se identifica como uno de los dos grupos más vulnerables en salud mental a las personas con psicopatología previa, debido a que muchos han sufrido la incomunicación al mundo exterior. Ante ello, se plantea la injerencia de la teleasistencia en ciertas psicopatologías, con el fin de resguardar su bienestar psicológico durante la pan- demia en el país; lo cual representa un reto para el sistema de salud nacional (Inchausti et al., 2020). De ese modo, es necesario realizar un seguimiento del tratamiento de los pacientes con psicopatologías, ya que podría deteriorarse la salud mental de forma progresiva (Correa et al., 2020; Alarcón, 2020).

Al analizar la situación de los pacientes hospitalizados en el centro de salud y la atención a sus allegados, se pudo observar que muchos de ellos sufrieron reacciones psicológicas negativas, siendo la ansiedad y depresión las más frecuentes, debido al inminente aislamiento que se encontraban atravesando, por lo que se implementaron formas complementarias de comunicación con sus familiares (Presa-García et al., 2021). Asimismo, se señala la posibilidad de que la salud mental en las personas con enfermedades psicológicas previas empeore tras estas medidas tomadas por distintos gobiernos (Reis da Silva et al., 2020). Estos autores tienen en cuenta como muy importante el hecho de mantengan comunicación con sus seres queridos.

Las cifras sobre las personas con enfermedades psicológicas durante el confinamiento son alarmantes con respecto al aumento de la ansiedad, depresión, trastorno de sueño, entre otros. Si bien el confinamiento social ha sido una de las medidas para evitar los contagios, también ha constituido una medida "destructiva" para la salud mental de quienes ya sufrían patologías mentales (Rodríguez et al., 2020).

\section{Salud mental en el personal de salud}

El personal sanitario que ha sido el grupo de mayor contacto y acercamiento con el covid-19, ha tenido como función principal cuidar la salud de quienes presentaban síntomas graves de la enfermedad. Sin embargo, 
a la par que trabajaban y cuidaban a pacientes, también tenían la responsabilidad de cuidar su salud y evitar la transmisión del virus (Becerra y Ybaseta, 2020; García-Iglesias et al., 2020). Esta situación compleja conllevó a que tuviesen dificultades frente a las distorsiones cognitivas que se presentaban al frente de situaciones nuevas y que puedan representar un peligro como el estrés y ansiedad. Inclusive, en el caso hipotético que un familiar cercano se enfermase, podrían sentir miedo, culpa, desánimo, frustración e impotencia que promueven emociones negativas que resultan ser perjudiciales para la salud. Aunado a ello, las renuncias de colegas y escases de personal asistencial complicaron la situación y estabilidad emocional del personal sanitario. De esa forma, se consideró necesario que tengan un tratamiento y seguimiento sobre su equilibrio cognitivo y conductual (López y López, 2020).

Los trabajadores sanitarios durante la pandemia han sufrido ataques de pánico, crisis de ansiedad, que en un futuro puede traer consecuencias postraumáticas debido al estrés y depresión bajo los cuales están sometidos y los pone en riesgo de cierta forma (Acosta-Quiroz y Iglesias-Osores, 2020). El personal sanitario (enfermas, trabajadores de salud, biólogos, médicos) también estuvo en riesgo de dañar su salud mental ya que, de por sí, el ambiente donde laboran está expuesto a un nivel alto de contagio. Esto influye en el equilibrio mental y, por lo tanto, es necesario que sean atendidos para prevenir alteraciones cognitivas y conductuales para que continúen en su labor (Torres-Muñoz et al., 2020). Es imprescindible su atención primaria en salud mental dado que su trabajo es intenso con pacientes enfermos de covid-19. Es por ello que se debe reducir el impacto en la salud mental del personal y brindarle el cuidado que requie- re ya que puede traer consigo riesgos personales. Por ende, es necesario el acompañamiento de un profesional de la salud mental para resguardar el equilibrio en su salud cognitiva (Ybaseta-Medina y Becerra-Canales, 2020).

De tal manera, se tienen ciertas consideraciones sobre la ansiedad ya que ha generado daños en la salud mental del personal de salud quienes se enfrentan a un gran riesgo de contagio por las largas jornadas laborales tratando de salvar la vida de personas contagiadas. Por ello, el personal de salud presentó síntomas ansiosos y depresivos debido al elevado grado de estrés (Huarcaya-Victoria, 2020).

Además de tener preocupación por el aumento de casos de coronavirus, también tiene que lidiar con el síndrome de Burnout que es lo que mejor describe a la situación por la que pasan los profesionales de salud debido a la alta demanda de trabajo y las exigencias en estos tiempos donde todo resulta ser muy complicado. Por lo que pueden tener agotamiento, estrés, ansiedad desequilibro en la fuerza, insomnio, ira, temor, negación, entre otros (Lozano-Vargas, 2020; Pérez et al., 2021; Galindo-Vazquez et al., 2020). Son muchos factores que agravan el desequilibrio emocional y mental como el bajo apoyo social, los horarios, jornadas de trabajo, la falta de equipos y el no poder ver a los que más quieren. Por ello es necesario que se le brinde la importancia que se requiere a este síndrome para prevenir futuras alteraciones y graves consecuencias en la salud mental (Juárez-García, 2020).

El impacto psicológico que ha tenido el covid-19 en los profesionales sanitarios se refleja en los altos niveles de estrés debido a la sobrecarga de trabajo y las condiciones en las que se encuentran y que ocasionan deses- 
tabilizar su salud. Además, se identificó que las personas que más sufrían de ansiedad eran las mujeres y profesionales mayores (Dosil et al., 2020). Ellas soportan una gran sobrecarga anímica al observar, percibir y vivenciar los casos graves y múltiples muertes de enfermos; por lo tanto, su ansiedad y estrés puede volverse patológico debido a la sobrecarga psicológica que produce experiencias similares.

El proceso de afrontar el estrés, miedo, depresión y ansiedad ha sido complicado y sigue siéndolo debido a la incertidumbre de no saber si la situación mejorará o empeorará. El profesional encargado de la salud mental de enfermeros, técnicos y médicos deben enseñar a utilizar estrategias de afrontamiento para que sea la herramienta apropiada para enfrentar las crisis emocionales. No obstante, el personal de salud tiene poca disposición de tiempo para llevar a cabo estas capacitaciones. Por ese motivo, se recomienda poseer un grupo de apoyo (amigos o familia), de esa forma, el impacto emocional será menor (Valero et al., 2020). De igual manera, es necesario que se identifiquen los síntomas a tiempo ya que pueden desencadenar enfermedades mentales (Ybaseta-Medina y Becerra-Canales, 2020). Esto podría contribuir a que uno mismo ayude a reconocer síntomas de que la salud mental puede estar en riesgo, y en el caso extremo se llegue a un aislamiento completo como en Argentina (Alomo et al., 2020; Eidman et al., 2020).

Las respuestas que se brinden en este tipo de situaciones tienen que basarse en el objetivo de alcanzar una mejora en la salud mental del personal sanitario o en prevenir que se vean expuestos al riesgo en situaciones como estas. Por lo cual, es fundamental la atención oportuna a aquellos trabajadores de salud que presenten problemas en cuanto a su salud mental y evaluarlos de tal manera que se apoyen entre sí durante el proceso (Muñoz et al., 2020). En síntesis, las investigaciones sobre el personal de salud evidencian la necesidad de atención a problemas ansiosos y depresivos en el proceso de evaluación de la salud mental.

\section{Estrategias para controlar la ansiedad des- de perspectivas institucionales internacio- nales}

En el área de la salud mental los profesionales como neurólogos, psiquiatras y psicólogos se han enfrentado al reto sobre el proceso de tratamiento, donde la virtualización ha sido la principal estrategia que a través de herramientas como las videoconferencias ha aportado en sobremanera a que el paciente y el terapeuta mantenga contacto. De ese modo, una estrategia fundamental ha sido la telesalud, el cual es una opción de mantener esa supervisión con el médico de confianza mediante herramientas virtuales, dispositivos informáticos en relación con el servicio de internet y las videollamadas como recurso de medida eficaz en diferentes situaciones médicas (Caetano et al., 2020).

Para el tratamiento de la ansiedad, estrés y depresión en el contexto covid-19, se priorizó los ejercicios físicos inter diarios (las prácticas físicas no deben tener como resultado dolor, sino una liberación de estrés), programar un horario de actividades (ocupaciones en el interior del hogar), establecer metas medibles, identificación y control de pensamientos irracionales, comunicación activa, definir una zona de confort autorregulada, utilizar vestuario cómodo, agregar indicadores de tiempo flexibles y organizar capacitaciones en dirección a la formación de un desarrollo emocional adecuado, tolerancia en el dialogo, entre otros (Faro et al., 2020; 
Lozano-Vargas, 2020; Urzúa et al., 2020).

\section{CONCLUSIONES}

El estudio abordó el tema de salud mental principalmente donde se cumplieron los criterios de la investigación que recogen 51 artículos de diferentes bases de datos. Debido al amplio contenido del tema, se profundizó sobre ciertas patologías como la ansiedad, estrés y depresión que se han presentado dentro del contexto de pandemia. Debido al aislamiento se incrementaron los casos de ansiedad y depresión en grupos como trabajadores, estudiantes escolares y universitarios, quienes mantenían irregularidades en sus hábitos. Además, el cambio repentino hacia la restricción social produjo que las personas tuvieran dificultades en su adaptación al trabajo remoto y educación sin contacto social.

Por otro lado, los pacientes que padecen de una patología mental se vieron afectados en sobre manera. Su tratamiento a través de los programas de salud mental tuvo que ser suspendido hasta que se adapten a un sistema virtual, para ejecutar las terapias y tratamientos médicos. El hecho de que los pacientes psiquiátricos y psicológicos tuvieran que suspender sus actividades de terapia supone una alteración del tratamiento que afecta negativamente al paciente. De esa manera, la desatención de estos pacientes pudo desencadenar que los problemas de salud mental se agraven e inclusive producir comorbilidad psiquiátrica.
Otro grupo de alto riesgo que presentó problemas en su salud mental ha sido el personal de salud en los hospitales, clínicas y postas médicas. Estos mantuvieron contacto directo con pacientes contagiados por el covid-19. Entre los problemas que presentaron en su salud mental se encontró el estrés, ansiedad y depresión. El primero estuvo relacionado a la tensión de atender a personas enfermas del virus y la disminución del personal sanitario lo que incrementó el trabajo de los que permanecían laborando. El segundo se relacionó con el pensamiento de que ellos pueden contraer la enfermedad y, a la par, transmitirlo a sus familiares o seres queridos. El tercero se vinculó al aislamiento que tuvieron de sus familiares y el extremo aislamiento de las amistades, lo que produjo un conjunto de pensamientos de tristeza y en ciertos casos conllevó a la depresión.

Por último, las estrategias utilizadas para contrarrestar los problemas de salud mental estuvieron relacionados con la virtualización, por ejemplo: telesalud, donde se usaron las videoconferencias para llevar a cabo las sesiones de terapia. Por otro lado, los profesionales de salud mental sugieren que el tratamiento no solo sea a nivel racional (cognitivo), sino mediante el ejercicio físico, cambio de rutinas o establecimiento de horarios programados para llevar un control sobre las actividades del día; y se esa forma, evitar una vida sedentaria que genere pensamiento distorsionados los cuales afectan la salud mental.

\section{REFERENCIAS BIBLIOGRÁFICAS}

Acosta-Quiroz, J., y Iglesias-Osores, S. (2020). Salud mental en trabajadores expuestos a COVID-19. Revista de Neuro-Psiquiatría, 83(3). https://doi.org/10.20453/rnp.v83i3.3784

Alarcón, R. (2020). Perspectivas éticas en el manejo de la pandemia COVID-19 y de su impacto en la salud mental. Revista de Neuro-Psiquiatría, 83(2). http://dx.doi.org/10.20453/rnp. v83i2.3757 
Alomo, M., Gagliardi, G., Peloche, S., Somers, E., Alzina, P., y Prokopez, C. (2020). Efectos psicológicos de la pandemia COVID-19 en la población general de Argentina. Revista de la Facultad de Ciencias Médicas de Córdoba, 77(3), 176-181. https://doi.org/10.31053/1853.0605. v77.n3.28561

Álvarez-Villaseñor, A. S., Flores-Osuna, U., Torres-Barrera, J. S., Flores-Torrecillas, R., Fuentes, C., Reyes, L. L., Barbosa, F. J., Chávez, M., Guzmán, B. G. y González, A. (2020). Ansiedad en presencia de alimentos en escolares de Baja California Sur. Nutrición Hospitalaria, 37(4), 692-697. https://dx.doi.org/10.20960/nh.03044

Bakr, H., Al-Sukayt, A., Chinna, K., Nurunnabi, M., Sundarasen, S., Kamaludin, K., Mohammad, G. y Abid, S. F. (2020). Anxiety Level of University Students During COVID-19 in Saudi Arabia. Frontiers in Psychiatry, 11, e579750. https://doi.org/10.3389/ fpsyt. 2020.579750

Barrera, A., Neira-Cofré, M., Raipán-Gómez, P., Riquelme-Lobos, P., y Escobar, B. (2019). Apoyo social percibido y factores sociodemográficos en relación con los síntomas de ansiedad, depresión y estrés en universitarios chilenos. Revista de Psicopatología y Psicología Clínica, 24(2), 105-115. https://doi.org/10.5944/rppc.23676

Becerra, B. y Ybaseta, J. (2020). Salud autopercibida en trabajadores de enfermería del primer nivel de atención durante la pandemia de Covid-19. Revista Médica PANACEA, 9(2). https://doi.org/10.35563/rmp.v9i2.330

Bueno-Notivol, J., Gracia-García, P., Olaya, B., Lasheras, I., López-Antón, R., y Santabárbara, J. (2020). Prevalence of depression during the COVID-19 outbreak: A meta-analysis of community-based studies. International Journal of Clinical and Health Psychology, 21(1). http://doi.org/10.1016/j.ijchp.2020.07.007

Caetano, R., Baptista, A., Carneiro, A. C., Nepomuceno, C. C., da Rocha, G., Lacerda, D., y Mendes, R. (2020). Desafios e oportunidades para telessaúde em tempos da pandemia pela COVID-19: uma reflexão sobre os espaços e iniciativas no contexto brasileiro. Cadernos de Saúde Pública, 36(5). https://doi.org/10.1590/0102-311X00088920

Correa, H., Malloy-Diniz, L., y da Silva, A. (2020). Why psychiatric treatment must not be neglected during the COVID-19 pandemic. Letters to the Editors, 42(4). https://doi. org/10.1590/1516-4446-2020-0995

De la Rosa, A., Moreyra, L., y De la Rosa, N. G. (2020). Intervenciones eficaces vía internet para la salud emocional en adolescentes: una propuesta ante la pandemia por COVID-19. Hamut'ay Revista de divulgación científica de la Universidad Alas Peruanas, 7(2), 18-33. http://dx.doi.org/10.21503/hamu.v7i2.2128 
Dos Santos, B., Scorsolini-Comin, F., y Barcellos, R. d. (2021). Ser docente en el contexto de la pandemia de COVID-19: reflexiones sobre la salud mental. Index de Enfermería, 29(3). http://scielo.isciii.es/scielo.php?pid=S1132-12962020000200008\&script=sci_arttext\&tln$\mathrm{g}=\mathrm{en}$

Dosil, M., Ozamiz-Etxebarria, N., Redondo, I., Alboniga-Mayor, J. J., y Picaza, M. (2020). Impacto psicológico de la COVID-19 en una muestra de profesionales sanitarios españoles. Revista de Psiquiatría y Salud Mental, 14(2), 106-112. http://doi.org/10.1016/j. rpsm.2020.05.004

Eidman, L., Arbizú, J., Lamboglia, A. T. y del Valle, L. (2020). Salud Mental y síntomas psicológicos en adultos argentinos de población general en contexto de pandemia por COVID-19. Subjetividad y Procesos Cognitivos, 24(2). https://publicacionescientificas.uces.edu.ar/index.php/subyprocog/article/view/967

Faro, A., de Andrade, M., de Cassia, T., Reis, C., Pereira, B. F., y Santos, L. (2020). COVID-19 e saúde mental: a emergência do cuidado. Estudios de Psicología, 37. https://doi.org/10.15 90/1982-0275202037e200074

Galindo-Vázquez, O., Ramírez-Orozco, M., Costas-Muñiz, R., Mendoza-Contreras, L., Calderillo-Ruíz, G., y Meneses-García, A. (2020). Síntomas de ansiedad, depresión y conductas de autocuidado durante la pandemia de COVID-19 en la población general. Gaceta Médica de México, 156, 298-305. http://doi.org/10.24875/GMM.20000266

García-Iglesias, J. J., Gómez-Salgado, J., Martín-Pereira, J., Fagundo-Rivera, J., Ayuso-Murillo, D., Martínez-Riera, J. R., y Ruiz-Frutos, C. (2020). Impacto del Sars- COV 2 (Covid-19) en la salud mental de los profesionales sanitarios: Una revisión sistemática. Revista Española de Salud Pública, 94, e202007088. https://www.mscbs.gob.es/biblioPublic/publicaciones/ recursos_propios/resp/revista_cdrom/VOL94/REVISIONES/RS94C_202007088.pdf

Gómez, M. I. (2020). Disminución de la ansiedad en las víctimas del bullying durante el confinamiento por el COVID-19. Revista de Educación a Distancia, 21(65). https://doi. org/10.6018/red.439601

González-Jaimes, N. L., Tejeda-Alcántar, A. A., Espinosa-Méndez, C. M., y Ontiveros-Hernádez, Z. O. (2020). Impacto psicológico en estudiantes universitarios mexicanos por confinamiento durante la pandemia por Covid-19. Preprint. https://doi.org/10.1590/SciELOPreprints. 756

González-Rodríguez, A. y Labad, J. (2020). Salud mental en tiempos de la COVID: reflexiones tras el estado de alarma. Medicina Clínica, 155(9), 392-394. http://doi.org/10.1016/j.medcli.2020.07.009 
Hernández-Rodríguez, J. (2020). Impacto de la COVID-19 sobre la salud mental de las personas. Revista Científica Villa Clara, 24(3), 578-594. http://scielo.sld.cu/pdf/mdc/v24n3/10293043-mdc-24-03-578.pdf

Herrera, I., Medina, I., Carillo, A., y Cortés, D. B. (2021). abordaje de enfermería sobre el miedo y ansiedad ante la muerte en personas adultas mayores. Health and Addictions, 21(1), 159170. https://doi.org/10.21134/haaj.v21i1.544

Huarcaya-Victoria, J. (2020). Consideraciones sobre la salud mental en la pandemia de Covid-19. Revista Peruana de Medicina Experimental y Salud Pública, 37(2), 327-334. https://doi.org/10.17843/rpmesp.2020.372.5419

Inchausti, F., García-Poveda, N., Prado-Abril, J., y Sánchez-Reales, S. (2020). La psicología clínica ante la pandemia COVID-19 en España. Clínica y Salud, 31(2), 105-107. https:// dx.doi.org/10.5093/clysa2020a11

Juárez-García, A. (2020). Síndrome de burnout en personal de salud durante la pandemia COVID-19: un semáforo naranja en la salud mental. Revista de la Universidad Industrial de Santander, 52(4). https://revistas.uis.edu.co/index.php/revistasaluduis/article/view/11528

Lacruz-Gascón, T., Moraleda-Merino, J., Graell-Berna, M., Villa-Asensi, J., y Sepúlveda-García, A. (2019). Asma de inicio en la infancia: Impacto en la personalidad y la psicopatología en una muestra de adolescentes. Revista de Psicopatología y Psicología Clínica, 24, 49-57. http://doi.org/10.5944/rppc.22633

Leiva, A. M., Nazar, G., Martínez-Sanguinetti, M. A., Pettermann-Rocha, F., Ricchezza, J., y Celiz-Morales, C. (2020). Dimensión psicosocial de la pandemia: la otra cara del Covid-19. Ciencia y Enfermería, 26(1). http://dx.doi.org/10.29393/ce26-3dpal60003

López, I., y López, I. (2020). La Salud Mental del personal sanitario ante la Pandemia del COVID-19. Enfermería Investiga, 6(1). https://revistas.uta.edu.ec/erevista/index.php/enfi/ article/view/1026/956

Lorenzo, J., Díaz, H., Cuello, M., Ordoñez, L y Díaz, J. (2021). Síntomas depresivos en pacientes diagnosticados con COVID-19 en Pinar del Río. Revista de Ciencias Médicas de Pinar del Río, 25(1), e4858. http://www.revcmpinar.sld.cu/index.php/publicaciones/article/ view/4858/pdf

Lozano-Vargas, A. (2020). Impacto de la epidemia del Coronavirus (COVID-19) en la salud mental del personal de salud y en la población general de China. Revista de Neuro-Psiquiatría, 83(1). http://dx.doi.org/10.20453/rnp.v83i1.3687 
Mazza, M. G., De Lorenzo, R., Conte, C., Poletti, S., Vai, B., Bollettini, I., Melloni, E. M. T., Furlan, R., Ciceri, F. y Rovere-Querini, P. (2020). Anxiety and depression in COVID-19 survivors: Role of inflammatory and clinical predictors. Brain, Behavior, and Immunity, 89, 594-600. https://doi.org/10.1016/j.bbi.2020.07.037

Medina, I. A., Carrillo, A. L., Pantoja, M., Torres, R., Medina, J. A., y Cortez, L. C. (2021). Influencia de la ansiedad y síntomas depresivos sobre la agencia de autocuidado en cuidadores de personas con enfermedad crónica. Health and Addictions, 21(1), 204-215. https:// doi.org/10.21134/haaj.v21i1.563

Merino-Navarro, D. y Díaz-Periánez, C. (2021). Prevención y tratamiento de la COVID-19 en la población pediátrica desde una perspectiva familiar y comunitaria: artículo especial. Enfermería Clínica, 31(1), 29-34. https://doi.org/10.1016/j.enfcli.2020.05.005

Motrico, E., Mateus, V., Bina, R., Felice, E., Bramante, A., Kalcev, G., . . Mesquita, A. (2020). Good Practices in Perinatal Mental Health suring the COVID-19 Pandemic: A Reportfrom Task-Force RISEUP-PPD COVID-19. Clínica y Salud, 31(3), 155-160. https://doi. org/10.5093/clysa2020a26

Muñoz, S. I., Molina, D., Ochoa, R., Sánchez, O., y Esquivel, J. A. (2020). Estrés, respuestas emocionales, factores de riesgo, psicopatología y manejo del personal de salud durante la pandemia por COVID-19. Acta Pediátrica de México, 41(451). http://dx.doi.org/10.18233/ APM41No4S1ppS127-S1362104

Ochoa, J. P., Ortiz, S. E., y Rojas, J. M. (2020). Lidiar con el estrés durante el brote de Covid-19. Revista Científica Mundo de la Investigación y el Conocimiento, 4(4), 144-151. https://doi. org/10.26820/recimundo/4.(4).octubre.2020.144-151

Organización Mundial de la Salud. (2020). Covid-19: cronología de la actuación de la OMS. https://www.who.int/es/news-room/detail/27-04-2020-who-timeline---covid-19

Ornell, F., Schuch, J., Sordi, A., y Paim, F. (2020). Pandemic fear and COVID-19: mental health burden and strategies. Brazilian Journal of Psychiatry, 42(3), 232-235. https://doi. org/10.1590/1516-4446-2020-0008

Ozamiz-Etxebarria, N., Dosil-Santamaria, M., Picaza-Gorrochategui, M., y Idoiaga-Mondragon, N. (2020). Niveles de estrés, ansiedad y depresión en la primera fase del brote del COVID-19 en una muestra recogida en el norte de España. Cadernos de Saúde Pública, 36(4), e00054020. http://doi.org/10.1590/0102-311X00054020 
Pech, D., Lira, J., Cruz, S. E. y Reynoso, L. (2021). Ansiedad, depresión, calidad de vida y consumo de tabaco en personas con diabetes mellitus tipo 2. Health and Addictions, 21(1). https://doi.org/10.21134/haaj.v21i1.526

Pérez, P. X., Noroña, D. R., y Vega, V. (2021). Repercusión SARS-CoV-2 en salud mental y bienestar psicológico del personal Centro de Salud Huambalo 2020. Revista Scientific, 6(19). https://doi.org/10.29394/Scientific.issn.2542-2987.2021.6.19.12.243-262

Presa-García, M., Pérez-Íñigo-Gancedo, J., Alonso-Martín, A., Muñoz-Cenjor, M., Iglesias-García, C., Abril-García, M., Martinez-Sanz, M., Robles-Bermejo, F., Ric-Benito, P., Fernandez-Faber, D., Suárez-Guinea, R., Rodriguez-Villarino, C., Juarez-Calvo, V., Rubio-Zabala, I. y Benavente-López, S. (2021). El Departamento de Psiquiatría y Salud Mental del Hospital Central de la Defensa $<<$ Gómez Ulla $>>$ durante la pandemia por SARS-CoV-2. Sanidad Militar, 76(2). https://scielo.isciii.es/pdf/sm/v76n2/1887-8571-sm-76-02-91.pdf

Ramírez-Ortiz, J., Castro-Quintero, D., Lerma-Córdoba, C., Yela-Ceballos, F., y Escobar-Córdoba, F. (2020). Consecuencias de la pandemia de la COVID-19 en la salud mental asociadas al aislamiento social. Colombian Journal of Anesthesiology, 48(4). https://doi. org/10.5554/22562087.e930

Reis da Silva, D. A., Weyll, R. F., y Conceição das Merces, M. (2020). Covid and the pandemic of fear: reflections on mental health. Revista de Saúde Pública, 54. https://doi.org/10.11606/ s1518-8787.2020054002486

Ribot, V. d., Chang, N., y González, A. L. (2020). Efectos de la COVID-19 en la salud mental de la población. Revista Habanera de Ciencias Médicas, 19(Supl.), e3307. https://dialnet. unirioja.es/servlet/articulo? codigo $=7445898$

Robles-Sánchez, J. (2020). La Psicología de Emergencias ante la COVID-19: Enfoque desde la Prevención, Detección y Gestión Operativa del Riesgo. Clínica y Salud, 31(2), 115-118. https://doi.org/10.5093/clysa2020a17

Rodríguez, N. S., Padilla, L. Á., Jarro, I. G., Suárez, B. I., y Robles, M. (2020). Factores de riesgo asociados a depresión y ansiedad por covid-19 (SARS-Cov-2). Journal of America Health, 4(1). https://doi.org/10.37958/jah.v4i1.64

Sánchez, I. M. (2021). Impacto psicológico de la COVID-19 en niños y adolescentes. Revista Médica de Santiago de Cuba, 25(1). http://www.medisan.sld.cu/index.php/san/article/ view/3245 
Sandín, B., Valiente, R., García-Escalera, J., Campagne, D., y Chorot, P. (2020). Impacto psicológico de la pandemia de COVID-19: Efectos negativos y positivos en poblacion española asociados al periodo de confinamiento nacional. Revista de Psicopatología y Psicología Clínica, 25(1), 1-21. https://doi.org/10.5944/rppc.28107

Saravia-Bartra, M. M., Cazorla-Saravia, P. y Cedillo-Ramirez, L. (2020). Nivel De Ansiedad De Estudiantes De Medicina De Primer Año De Una Universidad Privada Del Perú En Tiempos De Covid-19. Revista de la Facultad de Medicina Humana, 20(4). https://doi. org/10.25176/RFMH.v20i4.3198

Torres-Muñoz, V. T., Farias-Cortés, J. D., Reyes-Vallejo, L. A., y Guillén-Díaz-Barriga, C. G. (2020). Riesgos y daños en la Salud mental del personal sanitario por la atención a pacientes con COVID-19. Revista Mexicana de Urología, 80(3), 1-9. https://doi.org/10.48193/ rmu.v80i3.653

Tresáncoras, A., García-Oliva, C., y Piqueras, J. (2017). Relación del uso problemático de whatsapp con la personalidad y la ansiedad en adolescentes. Health and Addictions, 17(1), $27-$ 36. http://doi.org/10.21134/haaj.v17i1.272

Urrutía, G., y Bonfill, X. (2011). Declaración PRISMA: una propuesta para mejorar la publicación de revisiones sistemáticas y metaanálisis. Medicina Clínica, 135(11), 507-211. http:// doi.org/10.1016/j.medcli.2010.01.015

Urzúa, A., Vera-Villarroel, P., Caqueo-Urízar, A., y Polanco-Carrasco, R. (2020). La Psicología en la prevención y manejo del COVID-19. Aportes desde la evidencia inicial. Terapia Psicológica, 38(1). http://dx.doi.org/10.4067/S0718-48082020000100103

Valero, N. J., Vélez, M. F., Duran, Á. A., y Torres, M. (2020). Afrontamiento del COVID-19: estrés, miedo, ansiedad y depresión. Enfermería Investiga, 5(3), 63-70. https://revistas.uta. edu.ec/erevista/index.php/enfi/article/view/913/858

Ybaseta-Medina, J., y Becerra-Canales, B. (2020). El personal de Salud en la Pandemia por COVID-19. Revista Médica Panacea, 9(2). https://doi.org/10.35563/rmp.v9i2.322 